\title{
EL COSTE ENERGÉTICO DE LA DESALINIZACIÓN EN EL PROGRAMA A.G.U.A.
}

\author{
Alberto del Villar García \\ Departamento de Economía y Dirección de Empresas \\ Universidad de Alcalá
}

\section{RESUMEN}

Tras la derogación del trasvase del Ebro por el Real Decreto 2/2004 y la promulgación de la Ley 11/2005 de modificación del Plan Hidrológico Nacional, se reorientó la política del agua para satisfacer las nuevas necesidades de agua en el arco mediterráneo peninsular por medio de la desalinización. Esta política se ha centrado en un conjunto de actuaciones a través del llamado Programa A.G.U.A. (Actuaciones para la Gestión y Utilización del Agua). Las actuaciones en materia de desalinización de dicho programa se han materializado en la construcción de 16 nuevas plantas desalinizadoras con una capacidad de producción de casi $350 \mathrm{hm}^{3}$.

Del conjunto de estas actuaciones, al menos 6 de ellas pretendían satisfacer, de manera parcial, las necesidades de agua de unas 244.000 hectáreas de regadío, con un volumen de unos $155,37 \mathrm{hm}^{3}$. En 2008, el coste por metro cúbico estimado de producción, en las seis plantas destinadas a riego agrícola ascendía a 0,8634 €. El coste energético suponía aproximadamente un 52\% de los costes de operación y mantenimiento, y un $39 \%$ de los costes totales $\left(0,3373 € / \mathrm{m}^{3}\right)$. Este coste se estimó con una factura energética que recogía un precio de la energía en el entorno de 0,08 €/kwh. En 2012 el precio de la energía se sitúa por encima de $0,14 € / \mathrm{kwh}$, un $65 \%$ superior al calculado para 2008 . Este incremento se traduce en un aumento de la factura por esta fuente de recursos calculado en unos $0,21 € / \mathrm{m}^{3}$.

Palabras clave: Desalinización, coste energético, Programa AGUA.

\section{ABSTRACT}

\section{Energy Cost of Desalination in the A.G.U.A. Program}

Water Policy was reoriented in 2005, after Ebro Water Transfer abolition, to meet new water needs in the Mediterranean area through desalination. This policy has focused on a series of measures across the program called AGUA (Measures for the Management and Use of Water, in Spanish acronym). This Program has materialized in the construction of 16 new desalination plants with an average production about $350 \mathrm{Mm}^{3}$.

At least 6 of these plants covers part of the water needs to 244,000 ha, with an average water production about $155.37 \mathrm{Mm}^{3}$. In 2008, Average water production cost was estimated about $€ 0.8634$. Energy costs suppose nearly $52 \%$ of operating and maintenance costs, and $39 \%$ of total cost (€ 0.3373$)$. This cost was estimated from a low cost energy ( $0.08 € / \mathrm{kwh})$. In 2012, energy prices are located at $0.14 € / \mathrm{kwh}$, about $65 \%$ up from the estimated in 2008 . This energy fare increase supposes a water average cost increase about $€ 0.21$.

Keywords: Desalination, energy cost, AGUA Program.

\section{INTRODUCCIÓN}

Tras la derogación del trasvase del Ebro por el Real Decreto 2/2004 y la promulgación de la Ley 11/2005 de modificación del Plan Hidrológico Nacional, se reorientó la política del agua para satisfacer las nuevas necesidades de agua en el arco mediterráneo peninsular por medio de la desalinización.

Contacto: alberto.delvillar@uah.es 
Esta política se ha centrado en un conjunto de actuaciones a través del llamado Programa A.G.U.A. (Actuaciones para la Gestión y Utilización del Agua). Las actuaciones en materia de desalinización de dicho programa se han materializado en la construcción de 17 nuevas plantas desalinizadoras con una capacidad de producción de casi $350 \mathrm{hm}^{3}$. Del conjunto de estas actuaciones, al menos 6 de ellas pretendían satisfacer, de manera parcial, las necesidades de agua de unas 244.000 hectáreas, con un volumen de unos $155,37 \mathrm{hm}^{3}$.

A través de la información obtenida de los Informes de Viabilidad de Proyectos, establecidos a partir de la Ley $11 / 2005$, es posible acceder a realizar un análisis financiero referente a los proyectos hidráulicos financiados desde el Ministerio de Medio Ambiente ${ }^{1}$. En total, se pueden analizar unos 16 proyectos que se han aprobado en los últimos años referidos a esta tipología.

La cuantía de la inversión es importante ascendiendo a unos 1.119 millones de Euros presupuestados, 68,7 millones de Euros de media por cada instalación. La población y superficie de riego afectada es importante, teniendo destino a servicios de abastecimiento urbano el $54 \%$ de los volúmenes desalinizados $\left(189,1 \mathrm{hm}^{3}\right)$, siendo el $46 \%$ restante para usos en regadíos $\left(155,3 \mathrm{hm}^{3}\right)$.

Tabla 1. Información y unidades físicas de los proyectos de desalinización

\begin{tabular}{|c|c|c|c|}
\hline NOMBRE ACTUACIÓN (IVP) & Población & $\begin{array}{c}\text { Superficie } \\
\text { Riego }\end{array}$ & Volumen \\
\hline Desalinizadora Torrevieja (ATS) (Riego) & 2.400 .000 & 125.000 & 80.000 .000 \\
\hline Desalinizadora Bajo Almanzora (Almería) & 139.812 & 24.000 & 20.000 .000 \\
\hline Desalinizadora de Telde (Canarias) & 93.645 & 0 & 5.600 .000 \\
\hline Desalinizadora de Andratx (Mallorca) & 526.454 & 0 & 4.900 .000 \\
\hline Desalinizadora de Valdelentisco (Murcia) & 1.294 .694 & 6.000 & 70.200 .000 \\
\hline Desalobradora de Adra (Almería) & 23.195 & 0 & 3.380 .000 \\
\hline Desalinizadora Bahía de Alcudia (Mallorca) & 48.636 & 0 & 4.900 .000 \\
\hline Desalación Campo de Dalías (Almería) & 164.592 & 15.715 & 30.000 .000 \\
\hline Acondicionamiento Desalinizadora de Roque Prieto (Guía) & 55.203 & - & - \\
\hline Desalinizadora de Oropesa del Mar (Castellón) & 41.226 & 0 & 16.000 .000 \\
\hline Desalinizadora de Sagunto (Camp de Morvedre) & 62.500 & 0 & 8.000 .000 \\
\hline Ampliación Desalinizadora de El Mojón (Murcia) & 0 & 41.562 & 5.870 .000 \\
\hline Ampliación Desalinizadora de Águilas (Murcia) & 130.858 & 31.500 & 40.000 .000 \\
\hline Desalinizadora de Denia (Marina Alta) & 40.601 & 0 & 8.400 .000 \\
\hline Desalinizadora de Moncófar (Castellón) & 52.135 & 0 & 11.680 .000 \\
\hline Desalinizadora de Mutxamel (Alicante) & 443.261 & 0 & 17.500 .000 \\
\hline \multirow[t]{2}{*}{ Desalinizadora de la Costa del Sol (Málaga) } & 766.050 & 0 & 18.250 .000 \\
\hline & 6.282 .862 & 243.777 & 344.680 .000 \\
\hline
\end{tabular}

Fuente: Informes de Viabilidad de Proyectos (Artículo 46.5 Ley de Aguas). Elaboración propia. Cifras en habitantes, hectáreas y metros cúbicos.

A partir de la información contenida en la documentación pública disponible, se obtienen unos indicadores referidos a los costes de producción y de sus distintos componentes. Estos datos se utilizarán para analizar de manera independiente alguna de estas partidas, con la finalidad de efectuar un análisis de sensibilidad.

El objetivo pretendido es determinar el alcance de ciertas políticas sectoriales sobre actuaciones que desarrollan un sector estratégico de la economía española, e inciden en la incertidumbre de los procesos de toma de decisiones.

La razón fundamental estriba en comprobar cómo algunas políticas de corte trasversal presentan incidencias significativas sobre sectores estratégicos con alcance dispar en regiones que pueden tener una cierta dependencia estratégica de algún recurso productivo (caso del agua o energía).

\section{1 http://www.magrama.gob.es/es/agua/planes-y-estrategias/informes-de-viabilidad-de-obras-hidraulicas/}


Este trabajo se estructura en tres partes. En primer lugar se analizan los costes de diseño de las instalaciones dedicadas a los procesos de desalinización, desde una óptica centrada en la evolución de la tecnología y los rendimientos energéticos. A continuación se analiza el coste de las actuaciones del Programa A.G.U.A. en materia de desalinización, contemplando la estructura de costes de las 16 instalaciones sobre las que se dispone de una cierta información detallada. Por último, se acomete un análisis de sensibilidad sobre esta estructura de costes a partir de uno de sus componentes, considerado el más incierto y volátil, como es el coste energético.

\section{EL COSTE DE LAS ACTUACIONES EN MATERIA DE DESALINIZACIÓN}

En este apartado se van a tratar los distintos elementos que conforman los costes financieros de producción y explotación de las plantas desalinizadoras instaladas en España. Para ello, se analizará en primer término el estado y evolución de la cuestión de la desalinización para, a continuación, analizar los costes de los proyectos de esta naturaleza que han pasado el trámite de análisis de viabilidad previsto en el Artículo 46.5 de la Ley de Aguas.

\subsection{Evolución de la desalinización}

Existen muchas referencias respecto de los costes de la desalinización en los últimos años en España. Se ha analizado la evolución de estos costes en numerosos trabajos y con diferentes criterios.

Generalmente las variables y datos recogidos en estos análisis no se refieren a costes propiamente de los procesos y de las actuaciones. Los indicadores ofrecidos internacionalmente y a nivel nacional se refieren a precios de adjudicación y de concesiones, que no incluyen la parte que es financiada por fondos de ayuda al desarrollo, fondos europeos, financiación pública estatal, y otra no cubierta a través de las tarifas.

En las últimas décadas los costes medios por unidad de agua producida (metro cúbico) en las plantas desalinizadoras ha descendido a la par que lo hacía el consumo energético. El consumo energético es una parte fundamental del coste del proceso de desalación y su reducción se asocia con la disminución del coste de producción.

Desde 1970, año de instalación de la primera planta de producción de agua potable a partir de agua salada (Lanzarote), la evolución del coste ha ido ligada a la mejora tecnológica y reducción del consumo energético.

Tabla 2. Evolución del coste unitario de producción de agua a través de desalinizadoras

\begin{tabular}{|c|c|c|}
\hline Año & $\mathrm{Kwh} / \mathrm{m}^{3}$ & $€ / \mathbf{m}^{3}$ \\
\hline 1970 & 22 & 2,1 \\
\hline 1980 & 18 & 1,8 \\
\hline 1985 & 15 & 1,11 \\
\hline 1988 & 13 & 1,1 \\
\hline 1989 & 8,5 & 0,96 \\
\hline 1990 & 6,2 & 0,75 \\
\hline 1992 & 5,8 & 0,72 \\
\hline 1992 & 5,5 & 0,69 \\
\hline 1996 & 5,3 & 0,66 \\
\hline 1998 & 4,8 & 0,52 \\
\hline 1999 & 4,5 & 0,52 \\
\hline 2000 & 4,0 & 0,5 \\
\hline 2001 & 3,7 & 0,49 \\
\hline 2002 & 3,5 & 0,48 \\
\hline 2003 & 3,3 & 0,46 \\
\hline
\end{tabular}

Fuente: Etxaniz, J. (2005), Curso de Desalación AEDyR. 
Si en 1970 se precisaban 22 kilovatios hora para producir un metro cúbico de agua desalinizada, en 2003 este cociente había descendido hasta 3,3 kilovatios hora por metro cúbico². Con ello, el coste energético, y con ello el coste de producción por unidad de agua (metro cúbico), se ha ido reduciendo paulatinamente a lo largo de las últimas décadas hasta llegar a estas estimaciones de 0,46 €/ $\mathrm{m}^{3}$, e incluso inferiores a esta cifra.

No solamente los costes energéticos se han reducido en los últimos años, también otros costes como los de reposición de membranas y mantenimiento han acompañado esta tendencia a la baja, lo que unido a una progresiva reducción de los costes de inversión por unidad instalada y búsqueda de mayores economías de escala que se traducen en una repercusión vía amortizaciones por unidad producida cada vez menor.

En 2004, el Centro de Estudios Hidrográficos (CEDEX) realizó un análisis de los costes de desalación con previsiones basadas en grandes instalaciones (superiores a una capacidad de más de $60.000 \mathrm{~m}^{3} / \mathrm{día}$ ) a plena ocupación y con precios de la energía estables. Los resultados de estos estudios sirvieron de base para poner en marcha el proyecto de desalinización generalizada del Programa A.G.U.A., dado que , según estos estudios, los costes de producción unitarios podían alcanzar valores inferiores a $0,40 €$ por metro cúbico, tal y como se refleja en la tabla 3.

Tabla 3. Evolución de los costes del agua desalinizada

\begin{tabular}{|l|c|c|c|c|c|}
\hline & Unidades & 1995 & 2002 & 2004 & 2010 \\
\hline 1. Bases de cálculo & & & & & \\
\hline Coste de inversión & $€ / \mathrm{m}^{3}-$ día & 890 & 610 & 600 & 590 \\
\hline Amortización & Años & 15 & 15 & 15 & 15 \\
\hline Interés & $\%$ & 10 & 4 & 4 & 4 \\
\hline Consumo energía & $\mathrm{kWh} / \mathrm{m}^{3}$ & 5,3 & 4,1 & 3,6 & 2,9 \\
\hline Precio energía & $€ / \mathrm{kWh}$ & 0,077 & 0,048 & 0,048 & 0,048 \\
\hline 2. Costes parciales & & & & & \\
\hline Energía & $€ / \mathrm{m}^{3}$ & 0,408 & 0,196 & 0,172 & 0,139 \\
\hline Personal & $€ / \mathrm{m}^{3}$ & 0,036 & 0,036 & 0,030 & 0,025 \\
\hline Productos Químicos & $€ / \mathrm{m}^{3}$ & 0,030 & 0,028 & 0,028 & 0,030 \\
\hline Mantenimiento & $€ / \mathrm{m}^{3}$ & 0,024 & 0,024 & 0,024 & 0,024 \\
\hline Membranas & $€ / \mathrm{m}^{3}$ & 0,018 & 0,018 & 0,016 & 0,014 \\
\hline TOTAL EXPLOTACIÓN & $€ / \mathrm{m}^{3}$ & 0,516 & 0,302 & 0,270 & 0,232 \\
\hline TOTAL AMORTIZACIÓN & $€ / \mathrm{m}^{3}$ & 0,337 & 0,170 & 0,168 & 0,165 \\
\hline COSTE TOTAL & $€ / \mathrm{m}^{3}$ & 0,853 & 0,472 & 0,438 & 0,397 \\
\hline
\end{tabular}

Fuente: Torres (2004). Agua de mar, toma abierta, entrega en cota 100 a $15 \mathrm{Km}$.

Otros autores, en fechas más recientes, corroboran estos cálculos señalando costes de explotación por debajo del umbral de $0,45 € / \mathrm{m}^{3}$. Es el caso de Estevan (2008), quien señala que en términos actuales se puede estimar que los costes de explotación unitarios, sin incluir costes de inversión, para una planta de una capacidad alrededor de 30.000 metros cúbicos diarios, por medio de la tecnología de ósmosis inversa, a plena ocupación anual, se sitúa en una horquilla comprendida entre los $0,35 € / \mathrm{m}^{3}$ y los $0,45 € / \mathrm{m}^{3}$. Estas cifras, como es lógico, dependiendo de factores diversos como es el punto de entrega, tamaño de la planta, condiciones de la toma, etc.

Como se indica en el trabajo de Estevan (2008: 5-6), los costes de entrega pueden llegar a distorsionar en cierta forma los niveles de costes unitarios. Estos costes no proceden, en justicia, de los procesos de desalinización. Son costes de transporte como los de cualquier otra estación potabilizadora que, siguiendo el criterio de estandarización propuesto por el CEDEX ${ }^{3}$, oscilan entre $0,04 € / \mathrm{m}^{3}$ y $0,06 € / \mathrm{m}^{3}$.

$2 \mathrm{Al}$ menos en términos de ensayos de laboratorio. Existe un límite en la tecnología actual para el bombeo a alta presión que se cifra en unos $2,9 \mathrm{kWh} / \mathrm{m}^{3}$, por debajo de este límite no resulta posible vencer la presión osmótica. A esta energía hay que añadir otros bombeos e impulsiones para captar el agua y distribuirla. Los consumos específicos de energía de las plantas de producción pueden ser reducidos a partir de este momento con mecanismos de recuperación energética.

3 Entrega en cota $100 \mathrm{~m}$. y a $15 \mathrm{Km}$. de la planta. 
Otro aspecto a considerar es el relativo a los costes de inversión y su cómputo. En el trabajo de Estevan (2008: 10-12) se analizan estos costes en función del período de cómputo de la amortización o de la vida útil de los elementos de la planta desalinizadora. En proyectos concretos, con un cómputo de la vida útil de la obra civil de la planta de 30 años y plazos inferiores para los distintos equipos e instalaciones, la repercusión sobre el coste unitario se estimaba en un valor comprendido entre $0,126 € / \mathrm{m}^{3}$ y $0,156 € / \mathrm{m}^{3}$.

Los resultados finales podrían arrojar un coste unitario comprendido entre $0,45 € / \mathrm{m}^{3}$ y $0,50 € / \mathrm{m}^{3}$. Valores que incorporan todos los costes y que no incluirían subvenciones ni ayudas financieras, de acuerdo a los datos analizados por Estevan.

Bajo estos cálculos, en 2005 se procedió a la formulación de un plan de desalinización masiva en el mediterráneo español que incluía unas 16 actuaciones de gran envergadura con una capacidad de producción de casi 350 hectómetros cúbicos anuales y una inversión de más de 1.000 millones de Euros, como parte de la sustitución de caudales que hubieran procedido del recientemente derogado Trasvase del Ebro.

\subsection{El coste de la desalinización en el Programa A.G.U.A.}

A la luz del planteamiento expuesto en el apartado anterior, resultan comprensibles ciertas decisiones en materia de política de aguas que se tomaron a principios de la década pasada.

Resolver un problema estructural de agua, que afectaba en mayor medida a las regiones del arco mediterráneo español, por medio de la instalación de grandes plantas desalinizadoras, se antojaba con estos datos como una buena alternativa económica y financiera frente a la resolución que se podía plantear por medio de transferencias de recursos hídricos de otras cuencas.

En estas circunstancias la derogación del Trasvase del Ebro dio paso a un ambicioso programa de desalinización masiva, incluido dentro de lo que se denominó Programa A.G.U.A.

De forma casi paralela, como hito reciente de transparencia en la Administración, a partir de 2006 los proyectos acometidos con financiación estatal han de estar sometidos a un informe de viabilidad de proyectos conforme señala el artículo 46.5 del Texto Refundido de la Ley de Aguas. Este documento ha de ser de exposición pública ${ }^{4}$ y proporciona información bastante detallada sobre los proyectos y actuaciones en materia de desalinización que se someten al procedimiento de declaración de interés general.

Cabe destacar la imposibilidad de diferenciar, si es que existen en dichas actuaciones y han sido tenidos en cuenta, los costes de entrega y puesta a disposición de los que pertenecen solamente al apartado de producción de agua desalinizada.

La anterior afirmación deriva de la documentación (ACUAMED, 2006) a la que se ha tenido acceso sobre el cálculo de costes referida al consumo energético. En dicha documentación se indica un consumo energético por unidad producida $\left(\mathrm{m}^{3}\right)$ de $3,8 \mathrm{kwh}$ a pie de planta. Coincidente con otros trabajos, que desglosan el consumo energético de una instalación de ósmosis inversa en la siguiente relación (Martínez et al, 2000):

$\begin{array}{ll}\text { Bombeo agua de mar: } & 0,2 \mathrm{Kwh} / \mathrm{m}^{3} \\ \text { Bombeo intermedio: } & 0,3 \mathrm{Kwh} / \mathrm{m}^{3} \\ \text { Bombeo alta presión: } & 3,3 \mathrm{Kwh} / \mathrm{m}^{3} \\ \text { Bombeo producto (a } 300 \text { m.s.n.m.): } & 1,3 \mathrm{Kwh} / \mathrm{m}^{3} \\ \text { Total: } & 5,1 \mathrm{Kwh} / \mathrm{m}^{3}\end{array}$

El volumen de inversión es considerable, alcanzando para unos 17 proyectos en materia de desalinización (16 nuevas plantas o ampliaciones), alrededor de los 1.100 millones de Euros.

Los costes anuales de conservación y mantenimiento, estimados en los proyectos de cada una de las actuaciones, ascienden a unos 216 millones de euros anuales. Esta cifra ha tenido que calcularse en la mayor parte de los documentos, dado que los valores reflejados en los informes contenían algunas distorsiones que dificultaban los cálculos correctos de estas partidas ${ }^{5}$.

\footnotetext{
4 Se publican en la página WEB del Ministerio de Agricultura y Medio Ambiente.

5 La mayor parte de los documentos distingue cada uno de los componentes de los costes de conservación y mantenimiento. No obstante, a la hora de determinar estos costes se recoge el valor actualizado de cada partida correspondiente a un período de explotación de 25 años. Posteriormente, para determinar su coste anual equivalente (CAE) se divide esta partida entre 25. Este proceder es técnicamente erróneo y no tiene en cuenta los procesos de valoración financiera. Lo correcto sería aplicar una fórmula financiera de valoración anual de esta partida partiendo del valor actual de los flujos de la misma. Esto es emplear fórmulas financieras que consideren el efecto del descuento financiero y el tipo de interés aplicable. Tan sólo se aplica este criterio en el $25 \%$ de los proyectos que se presentan.
} 
Tabla 4. Principales magnitudes plantas desalinizadoras

\begin{tabular}{|l|c|c|c|}
\hline \multicolumn{1}{|c|}{ NOMBRE ACTUACIÓN (IVP) } & Inversión & Costes C\&M & Volumen \\
\hline Desalinizadora Torrevieja (ATS) (Riego) & 287.087 .104 & 56.885 .454 & 80.000 .000 \\
\hline Desalinizadora Bajo Almanzora (Almería) & 43.639 .619 & 12.132 .495 & 20.000 .000 \\
\hline Desalinizadora de Telde (Canarias) & 11.811 .265 & 1.839 .281 & 5.600 .000 \\
\hline Desalinizadora de Andratx (Mallorca) & 14.428 .093 & 1.788 .418 & 4.900 .000 \\
\hline Desalinizadora de Valdelentisco (Murcia) & 141.454 .045 & 26.631 .099 & 70.200 .000 \\
\hline Desalobradora de Adra (Almería) & 12.897 .953 & 1.459 .775 & 3.380 .000 \\
\hline Desalinizadora Bahía de Alcudia (Mallorca) & 21.847 .973 & 2.093 .280 & 4.900 .000 \\
\hline Desalación Campo de Dalías (Almería) & 99.274 .102 & 22.039 .535 & 30.000 .000 \\
\hline Desalinizadora de Oropesa del Mar (Castellón) & 47.406 .007 & 12.022 .535 & 16.000 .000 \\
\hline Desalinizadora de Sagunto (Camp de Morvedre) & 27.478 .367 & 6.309 .265 & 8.000 .000 \\
\hline Ampliación Desalinizadora de El Mojón (Murcia) & 29.191 .813 & 3.835 .929 & 5.870 .000 \\
\hline Ampliación Desalinizadora de Águilas (Murcia) & 143.671 .118 & 25.099 .819 & 40.000 .000 \\
\hline Desalinizadora de Denia (Marina Alta) & 34.078 .740 & 6.391 .855 & 8.400 .000 \\
\hline Desalinizadora de Moncófar (Castellón) & 48.376 .235 & 7.986 .189 & 11.680 .000 \\
\hline Desalinizadora de Mutxamel (Alicante) & 69.848 .195 & 14.037 .884 & 17.500 .000 \\
\hline Desalinizadora de la Coste del Sol (Málaga) & 66.789 .657 & 15.116 .430 & 18.250 .000 \\
\hline TOTALES & 1.099 .280 .284 & 215.669 .240 & 344.680 .000 \\
\hline
\end{tabular}

Fuente: Informes de Viabilidad de Proyectos (Artículo 46.5 Ley de Aguas). Elaboración propia. Cifras en habitantes, hectáreas y metros cúbicos.

En el reparto de los costes de conservación y mantenimiento, los costes energéticos representan más de la mitad del total, seguidos por los costes de mantenimiento y reposición. Entre ambas partidas, engloban el $85 \%$ de los costes de conservación y mantenimiento de diseño anuales de estas plantas.

Figura 1. Estructura de los costes de conservación y mantenimiento

\section{Costes C\&M}

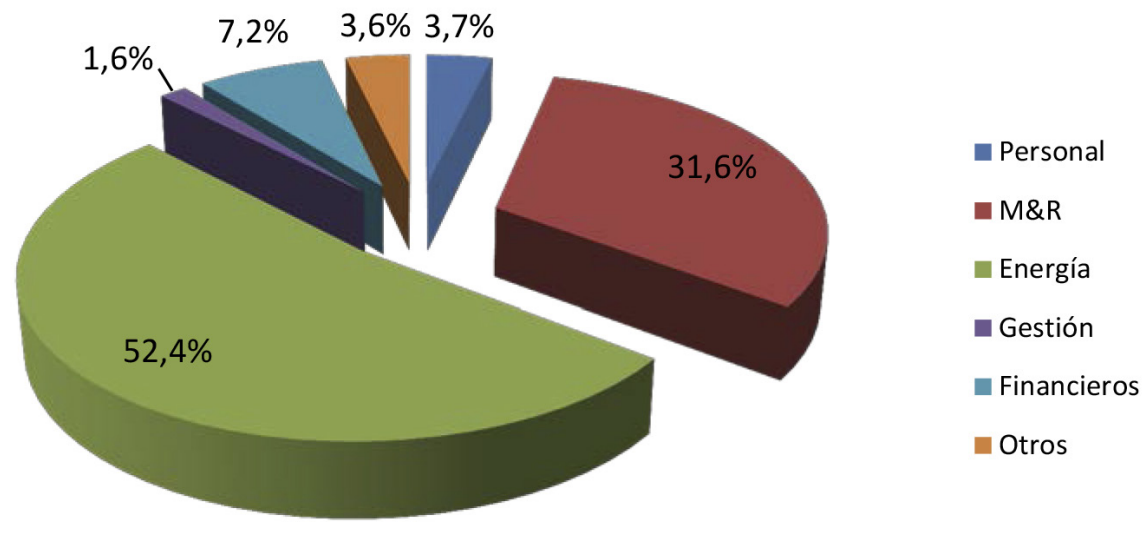

Fuente: Informes de Viabilidad de Proyectos (Artículo 46.5 Ley de Aguas). Elaboración propia.

Cabe destacar que, de acuerdo a estas cifras, el coste por metro cúbico de agua producida tendría unos costes medios de conservación y mantenimiento de diseño de unos 0,6257 €.

El cálculo de la anualidad de los costes de inversión presenta ciertas dificultades. La información suministrada en los Informes de Viabilidad de Proyectos de cada actuación señala el porcentaje de participación de la obra civil y de las instalaciones, por lo que se puede diferenciar diferentes períodos de vida útil para cada conjunto de elementos. 
No obstante, los períodos utilizados como referencia para el cálculo en los Informes de Viabilidad ascienden a 50 años, en el caso de la obra civil, y 25 para la maquinaria e instalaciones. Considerando dichos períodos como excesivos, se ha optado por calcular el valor de estos atendiendo a 25 años de vida útil para las inversiones relativas a la obra civil, y 10 años para las inversiones acometidas en instalaciones y maquinaria. De esta forma, los costes anuales de inversión estimados para el conjunto de las 16 plantas desalinizadoras ascienden a 98,13 millones de euros. Este importe proporciona un indicador de coste de inversión por metro cúbico producido de $0,2847 €$.

En su conjunto, los costes anuales de desalinización para la producción de 344,68 hm $\mathrm{hm}^{3}$ en las 16 plantas desalinizadoras que contempla el Programa A.G.U.A. asciende a 313,8 millones de euros. El coste unitario de desalación se cifra en unos $0,91 € / \mathrm{m}^{3}$ tomando como año base 2008 , que sería el año de entrada en funcionamiento de la mayor parte de las instalaciones.

Cabe reseñar que el valor medio determinado en los Informes de Viabilidad de Proyectos asciende a unos $0,5804 € / \mathrm{m}^{3}$, con valores comprendidos entre $0,4650 € / \mathrm{m}^{3}$ y $0,8282 € / \mathrm{m}^{3}$.

Tabla 5. Coste Anual Equivalente en las Plantas Desalinizadoras del Programa A.G.U.A.

\begin{tabular}{|l|c|c|c|c|c|c|}
\hline \multirow{2}{*}{ NOMBRE ACTUACIÓN (IVP) } & \multicolumn{3}{c|}{ Coste Anual Equivalente } & \multicolumn{3}{c|}{ Coste Anual Equivalente por $\mathbf{m}^{3}$} \\
\cline { 2 - 7 } & Inversión & C\&M & Total & Inversión & C\&M & Total \\
\hline Desalinizadora Torrevieja (ATS) (Riego) & 26.003 .912 & 56.885 .454 & 82.889 .366 & 0,3250 & 0,7111 & 1,0361 \\
\hline Desalinizadora Bajo Almanzora (Almería) & 4.757 .959 & 12.132 .495 & 16.890 .453 & 0,2379 & 0,6066 & 0,8445 \\
\hline Desalinizadora de Telde (Canarias) & 1.127 .147 & 1.839 .281 & 2.966 .428 & 0,2013 & 0,3284 & 0,5297 \\
\hline Desalinizadora de Andratx (Mallorca) & 1.540 .571 & 1.788 .418 & 3.328 .989 & 0,3144 & 0,3650 & 0,6794 \\
\hline Desalinizadora de Valdelentisco (Murcia) & 14.085 .902 & 26.631 .099 & 40.717 .001 & 0,2007 & 0,3794 & 0,5800 \\
\hline Desalobradora de Adra (Almería) & 1.115 .780 & 1.459 .775 & 2.575 .555 & 0,3301 & 0,4319 & 0,7620 \\
\hline Desalinizadora Bahía de Alcudia (Mallorca) & 1.752 .709 & 2.093 .280 & 3.845 .989 & 0,3577 & 0,4272 & 0,7849 \\
\hline Desalación Campo de Dalías (Almería) & 8.235 .397 & 22.039 .535 & 30.274 .931 & 0,2745 & 0,7347 & 1,0092 \\
\hline Desalinizadora de Oropesa del Mar (Castellón) & 4.188 .711 & 12.022 .535 & 16.211 .246 & 0,2618 & 0,7514 & 1,0132 \\
\hline Desalinizadora de Sagunto (Camp de Morvedre) & 2.397 .172 & 6.309 .265 & 8.706 .437 & 0,2996 & 0,7887 & 1,0883 \\
\hline Ampliación Desalinizadora de El Mojón (Murcia) & 2.501 .594 & 3.835 .929 & 6.337 .523 & 0,4262 & 0,6535 & 1,0796 \\
\hline Ampliación Desalinizadora de Águilas (Murcia) & 12.717 .364 & 25.099 .819 & 37.817 .183 & 0,3179 & 0,6275 & 0,9454 \\
\hline Desalinizadora de Denia (Marina Alta) & 2.857 .984 & 6.391 .855 & 9.249 .839 & 0,3402 & 0,7609 & 1,1012 \\
\hline Desalinizadora de Moncófar (Castellón) & 3.832 .884 & 7.986 .189 & 11.819 .073 & 0,3282 & 0,6837 & 1,0119 \\
\hline Desalinizadora de Mutxamel (Alicante) & 5.429 .520 & 14.037 .884 & 19.467 .404 & 0,3103 & 0,8022 & 1,1124 \\
\hline Desalinizadora de la Coste del Sol (Málaga) & 5.589 .780 & 15.116 .430 & 20.706 .210 & 0,3063 & 0,8283 & 1,1346 \\
\hline TOTALES & 98.134 .388 & 215.669 .240 & 313.803 .628 & 0,2847 & 0,6257 & 0,9104 \\
\hline
\end{tabular}

Fuente: Informes de Viabilidad de Proyectos (Artículo 46.5 Ley de Aguas). Elaboración propia. Cifras en Euros.

Teniendo en cuenta sólo las plantas e instalaciones que presentan una cierta dedicación para la producción de recursos de uso agrícola, el coste medio de desalinización de esas dos plantas ascendería a $0,8634 € / \mathrm{m}^{3}$. El volumen de producción de este tipo de recursos permitiría satisfacer aproximadamente un $15 \%$ de las necesidades de agua de los cultivos de regadío en el sureste peninsular.

\subsection{Los costes energéticos de la desalinización en el Programa A.G.U.A.}

Al respecto de algunas partidas y consideraciones sobre sus bases de cálculo, cabe destacar el capítulo correspondiente a los costes energéticos. Este capítulo de los costes está valorado a través de dos componentes. Por una parte, el término de potencia. Considerado un coste fijo de operación de las plantas, se aplica con independencia del nivel de consumo. Se deduce la aplicación de un valor fijo en 2006 de $9,992273 € / \mathrm{kW} / \mathrm{mes}^{6}$. Por otro lado, el término de consumo, considerando un precio de $0,045075 € / \mathrm{kWh}$, según las tarifas vigentes para 2006. Se considera un consumo de 3,8 $\mathrm{kWh}$ por cada metro cúbico de agua producido por las plantas.

6 Aplicando la tarifa general de larga duración 3.4 (Aprobada el 23 de diciembre de 2005). Esta tarifa ha sido sustituida a partir de 2008 por la entrada en vigor de la liberalización del mercado eléctrico. 
El cálculo del consumo energético dependerá de cada instalación de acuerdo a la potencia contratada, el volumen de producción y otros factores. Se puede estimar, a través de los parámetros de diseño de estas instalaciones, un consumo energético anual de 1.302.010 MWh, con un valor medio del kWh de 0,0867 €.

Tabla 6. Coste Anual Equivalente del Uso de Energía en las Plantas Desalinizadoras del Programa A.G.U.A.

\begin{tabular}{|c|c|c|c|c|}
\hline NOMBRE ACTUACIÓN (IVP) & Volumen $\left(\mathrm{m}^{3}\right)$ & $\begin{array}{l}\text { Energía } \\
(\mathrm{kWh})\end{array}$ & $\begin{array}{c}\text { Coste energía } \\
(€)\end{array}$ & $\begin{array}{c}\text { Coste energía } \\
(€ / \mathrm{kWh})\end{array}$ \\
\hline Desalinizadora Torrevieja (ATS) (Riego) & 80.000 .000 & 304.000 .000 & 29.217 .809 & 0,0961 \\
\hline Desalinizadora Bajo Almanzora (Almería) & 20.000 .000 & 76.000 .000 & 6.471 .512 & 0,0852 \\
\hline Desalinizadora de Telde (Canarias) & 5.600 .000 & 21.280 .000 & 1.088 .685 & 0,0512 \\
\hline Desalinizadora de Andratx (Mallorca) & 4.900 .000 & 18.620 .000 & 1.131 .931 & 0,0608 \\
\hline Desalinizadora de Valdelentisco (Murcia) & 70.200 .000 & 266.760 .000 & 14.199.766 & 0,0532 \\
\hline Desalobradora de Adra (Almería) & 3.380 .000 & $5.070 .000^{\mathrm{A}}$ & 271.555 & 0,0536 \\
\hline Desalinizadora Bahía de Alcudia (Mallorca) & 4.900 .000 & 18.620 .000 & 1.217 .078 & 0,0654 \\
\hline Desalación Campo de Dalías (Almería) & 30.000 .000 & 114.000 .000 & 12.766 .710 & 0,1120 \\
\hline Desalinizadora de Oropesa del Mar (Castellón) & 16.000 .000 & 60.800 .000 & 6.443 .959 & 0,1060 \\
\hline Desalinizadora de Sagunto (Camp de Morvedre) & 8.000 .000 & 30.400 .000 & 3.080 .249 & 0,1013 \\
\hline Ampliación Desalinizadora de El Mojón (Murcia) & 5.870 .000 & 22.306 .000 & 1.980 .325 & 0,0888 \\
\hline Ampliación Desalinizadora de Águilas (Murcia) & 40.000 .000 & 152.000 .000 & 11.627 .314 & 0,0765 \\
\hline Desalinizadora de Denia (Marina Alta) & 8.400 .000 & 31.920 .000 & 3.261 .938 & 0,1022 \\
\hline Desalinizadora de Moncófar (Castellón) & 11.680 .000 & 44.384 .000 & 4.330 .951 & 0,0976 \\
\hline Desalinizadora de Mutxamel (Alicante) & 17.500 .000 & 66.500 .000 & 7.868.018 & 0,1183 \\
\hline \multirow[t]{2}{*}{ Desalinizadora de la Coste del Sol (Málaga) } & 18.250 .000 & 69.350 .000 & 7.955 .577 & 0,1147 \\
\hline & 344.680 .000 & 1.302 .010 .000 & 112.913 .375 & 0,0867 \\
\hline
\end{tabular}

Fuente: Informes de Viabilidad de Proyectos (Artículo 46.5 Ley de Aguas). Elaboración propia. Se estima un consumo energético de 3,8 $\mathrm{kWh}$ por cada $\mathrm{m}^{3}$ de agua producida. ${ }^{\mathrm{A}}$ La planta Desalobradora se estima un consumo energético de $1,5 \mathrm{kWh} / \mathrm{m}^{3}$.

Esta partida tendrá vital importancia en el caso de evolucionar al alza los costes energéticos en el futuro. Como de hecho así ha ocurrido en los últimos años.

\section{ANÁLISIS DE SENSIBILIDAD DE LOS COSTES ENERGÉTICOS EN LAS PLANTAS DESALINIZADORAS}

La evolución del mercado energético en los últimos años no invita al optimismo respecto a la evolución del precio de la electricidad y la repercusión del coste de la energía sobre el coste de producción de agua desalinizada. A pesar de la liberalización del mercado de la electricidad el coste para las empresas por el suministro de energía ha experimentado un crecimiento medio de algo más del 64\% entre 2007 y 2012, habiendo alcanzado un importe medio por kWh para el primer semestre de 2012 de 0,1429 € (UNESA, 2013) ${ }^{7}$.

Este incremento en la factura energética para las instalaciones y plantas desalinizadoras supone una importante repercusión en el coste de producción. La energía supone un 48\% de los costes totales de producción, y un casi dos terceras partes de los costes de operación y mantenimiento. Lo que indica la especial repercusión que las variaciones en el precio de la energía tienen en estas plantas e instalaciones.

Tabla 7. Coste energético 2007 y 2012 en las Plantas Desalinizadoras del Programa A.G.U.A.

\begin{tabular}{|l|c|c|c|c|}
\hline \multirow{2}{*}{ NOMBRE ACTUACIÓN (IVP) } & \multicolumn{2}{|c|}{ Coste Energía (€) } & \multicolumn{2}{|c|}{ Repercusión Energía $\left(€ / \mathbf{m}^{3}\right.$ ) } \\
\cline { 2 - 5 } & 2007 & 2012 & 2007 & 2012 \\
\hline Desalinizadora Torrevieja (ATS) (Riego) & 29.217 .809 & 48.144 .735 & 0,3652 & 0,6018 \\
\hline Desalinizadora Bajo Almanzora (Almería) & 6.471 .512 & 10.663 .675 & 0,3236 & 0,5332 \\
\hline
\end{tabular}

7 Una diferencia de 0,0562 €/kWh entre ambos ejercicios. 


\begin{tabular}{|l|c|c|c|c|}
\hline Desalinizadora de Telde (Canarias) & 1.088 .685 & 1.793 .921 & 0,1944 & 0,3203 \\
\hline Desalinizadora de Andratx (Mallorca) & 1.131 .931 & 1.865 .181 & 0,2310 & 0,3806 \\
\hline Desalinizadora de Valdelentisco (Murcia) & 14.199 .766 & 23.398 .194 & 0,2023 & 0,3333 \\
\hline Desalobradora de Adra (Almería) & 271.555 & 447.464 & 0,0803 & 0,1324 \\
\hline Desalinizadora Bahía de Alcudia (Mallorca) & 1.217 .078 & 2.005 .486 & 0,2484 & 0,4093 \\
\hline Desalación Campo de Dalías (Almería) & 12.766 .710 & 21.036 .822 & 0,4256 & 0,7012 \\
\hline Desalinizadora de Oropesa del Mar (Castellón) & 6.443 .959 & 10.618 .273 & 0,4027 & 0,6636 \\
\hline Desalinizadora de Sagunto (Camp de Morvedre) & 3.080 .249 & 5.075 .596 & 0,3850 & 0,6344 \\
\hline Ampliación Desalinizadora de El Mojón (Murcia) & 1.980 .325 & 3.263 .154 & 0,3374 & 0,5559 \\
\hline Ampliación Desalinizadora de Águilas (Murcia) & 11.627 .314 & 19.159 .341 & 0,2907 & 0,4790 \\
\hline Desalinizadora de Denia (Marina Alta) & 3.261 .938 & 5.374 .980 & 0,3883 & 0,6399 \\
\hline Desalinizadora de Moncófar (Castellón) & 4.330 .951 & 7.136 .486 & 0,3708 & 0,6110 \\
\hline Desalinizadora de Mutxamel (Alicante) & 7.868 .018 & 12.964 .820 & 0,4496 & 0,7408 \\
\hline Desalinizadora de la Coste del Sol (Málaga) & 7.955 .577 & 13.109 .099 & 0,4359 & 0,7183 \\
\hline & 112.913 .375 & 186.057 .229 & 0,3276 & 0,5398 \\
\hline
\end{tabular}

Fuente: Informes de Viabilidad de Proyectos (Artículo 46.5 Ley de Aguas). Elaboración propia. Cifras comparando costes energía 2007 y 2012 (Fuente: UNESA).

En términos medios, el incremento de la factura energética ha supuesto un aumento de los costes medios de producción de agua desalinizada de algo más de $0,21 € / \mathrm{m}^{3}$, al pasar de un coste energético de $0,3276 € / \mathrm{m}^{3}$ a $0,5398 € / \mathrm{m}^{3}$. Aumento que representa un incremento del $23,3 \%$ en los costes de producción de 2012 respecto a los calculados en $2007^{8}$.

En el caso de las plantas e instalaciones dedicadas total o parcialmente a la producción de recursos para uso en actividades de riego, la situación es ligeramente mejor que para el conjunto de las instalaciones contempladas en el Programa A.G.U.A. El incremento medio de costes para estas plantas dedicadas al uso de regadío asciende a $0,20 € / \mathrm{m}^{3}$, alcanzando un coste de producción medio de $1,0641 € / \mathrm{m}^{3}$, frente al $1,1226 € / \mathrm{m}^{3}$ del conjunto de las instalaciones del Programa A.G.U.A.

Tabla 8. Coste producción agua desalinizada 2007 y 2012 en las Plantas Desalinizadoras del Programa A.G.U.A. dedicadas al uso de regadío

\begin{tabular}{|c|c|c|c|c|c|c|}
\hline \multirow{2}{*}{ NOMBRE ACTUACIÓN (IVP) } & \multirow{2}{*}{$\begin{array}{l}\text { Volumen } \\
\text { Total }\left(\mathrm{m}^{3}\right)\end{array}$} & \multirow{2}{*}{$\begin{array}{l}\text { Volumen } \\
\text { Riego }\left(\mathrm{m}^{3}\right)\end{array}$} & \multicolumn{2}{|c|}{ Costes 2007} & \multicolumn{2}{|c|}{ Costes 2012} \\
\hline & & & Total $(€)$ & por $\mathrm{m}^{3}$ & Total $(€)$ & por $\mathbf{m}^{3}$ \\
\hline Desalinizadora Torrevieja (ATS) (Riego) & 80.000 .000 & 60.000 .000 & 83.422 .792 & 1,0428 & 102.349 .719 & 1,2794 \\
\hline Desalinizadora Bajo Almanzora (Almería) & 20.000 .000 & 15.000 .000 & 16.544 .298 & 0,8272 & 20.736 .462 & 1,0368 \\
\hline Desalinizadora de Valdelentisco (Murcia) & 70.200 .000 & 37.000 .000 & 36.667 .611 & 0,5223 & 45.866 .039 & 0,6534 \\
\hline Desalación Campo de Dalías (Almería) & 30.000 .000 & 7.500 .000 & 31.472 .912 & 1,0491 & 39.743 .025 & 1,3248 \\
\hline Ampliación Desalinizadora de El Mojón (Murcia) & 5.870 .000 & 5.870 .000 & 6.323 .070 & 1,0772 & 7.605 .900 & 1,2957 \\
\hline Ampliación Desalinizadora de Águilas (Murcia) & 40.000 .000 & 30.000 .000 & 38.015 .085 & 0,9504 & 45.547 .112 & 1,1387 \\
\hline Totales & 246.070 .000 & 155.370 .000 & 212.445 .768 & 0,8634 & 261.848 .256 & 1,0641 \\
\hline
\end{tabular}

Fuente: Informes de Viabilidad de Proyectos (Artículo 46.5 Ley de Aguas). Elaboración propia.

El incremento de precio de la energía eleva de forma considerable los costes de producción. Por cada céntimo de euro que aumenta el precio del kWh, el coste de producción del agua desalinizada se incrementa de forma lineal en $0,038 € / \mathrm{m}^{3}$.

Todo el análisis anterior está basado en un pleno uso de la capacidad máxima de producción de las plantas desalinizadoras. Cabe pensar que ante situaciones de infrautilización de dicha capacidad de producción, las cifras resultantes serán más gravosas todavía para los usuarios. De hecho, si contemplamos

8 Todo ello manteniendo sin variación las restantes rúbricas de los costes. 
un análisis de sensibilidad sobre la capacidad de una planta, como es la Desalinizadora de Torrevieja, nos encontramos con cifras inesperadas.

Los costes fijos de inversión de la planta desalinizadora de Torrevieja ascienden a unos 26 millones de euros anuales, lo que unido a los costes fijos anuales de explotación (entendidos por aquella parte de los costes de explotación que no varían al alterarse la capacidad de producción) calculados en unos 21,7 millones de euros, nos dan como resultado unos costes fijos anuales de casi 48 millones de euros ${ }^{9}$. De acuerdo a la información proporcionada por el correspondiente Informe de Viabilidad del Proyecto de la Desalinizadora de Torrevieja, en 2007 el coste variable estimado era de unos $0,44 € / \mathrm{m}^{3}$. Con el incremento de precios de la energía acaecido desde 2007, en 2012 se calcula un coste variable unitario de unos $0,617 € / \mathrm{m}^{3}$. Teniendo en cuenta que la repercusión de los costes fijos es proporcional al empleo de la capacidad de producción de la planta, los costes unitarios de producción de agua desalinizada serán muy superiores a los previstos en la estrategia del Programa A.G.U.A. de hace casi una década.

Figura 2. Coste unitario de producción de la Desalinizadora de Torrevieja según capacidad de producción empleada

\section{Desalinizadora Torrevieja}

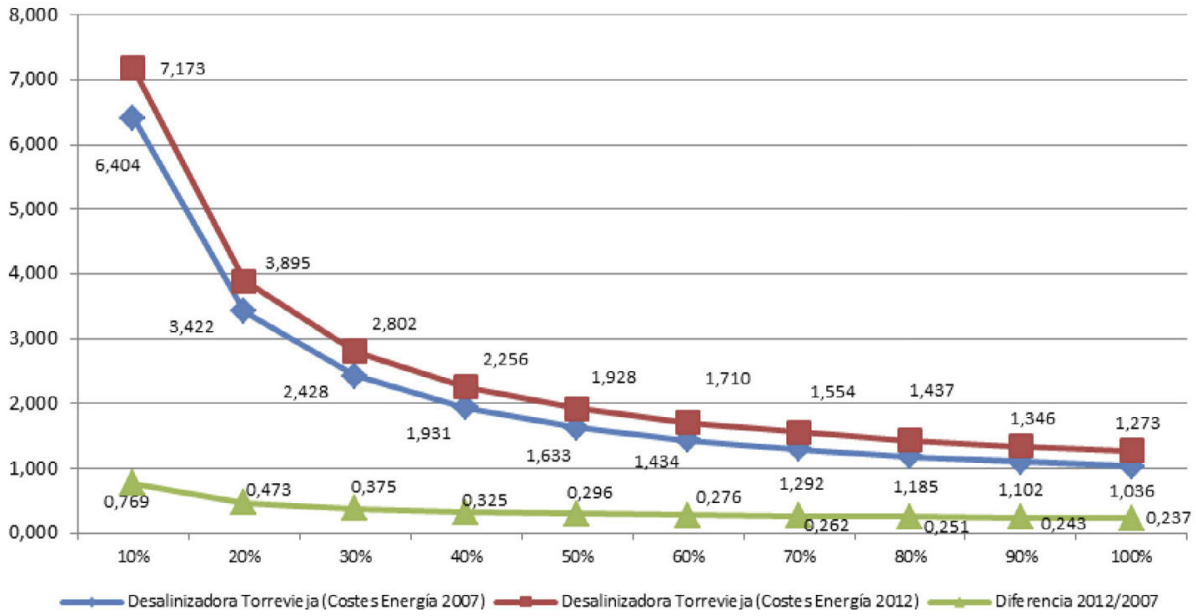

Fuente: Elaboración propia. Cifras en $€ / \mathrm{m}^{3}$

A partir de estos valores, y de acuerdo a las noticias disponibles sobre la actual capacidad de producción empleada (principios de 2014) en la planta de Torrevieja, en el entorno del 20-30\%, podemos señalar un coste presente de producción para esta planta de unos 2,8-3,9 euros por metro cúbico producido. Cifra que dista lejos del entorno de $0,40-0,60 € / \mathrm{m}^{3}$ que señalaban los informes y documentación de base con la que se proyectó el citado Programa A.G.U.A., que recogía la sustitución de caudales derivados del Ebro al sureste peninsular por caudales procedentes de plantas desalinizadoras. El ejemplo expuesto sobre la planta desalinizadora de Torrevieja es trasladable a otras plantas similares ubicadas en el Mediterráneo peninsular.

Estos niveles de costes nos hacen plantear nuevas preguntas acerca de la estrategia de asignación de recursos hídricos seguida en los últimos años. La primera deriva de la financiación de la construcción de las instalaciones. Tanto ACUAMED como la Mancomunidad de los Canales del Taibilla han experimentado un fuerte crecimiento de su endeudamiento con entidades de crédito. En el caso de la Mancomunidad de los Canales del Taibilla, sus deudas (tanto a corto como a largo plazo) se han multiplicado por cinco entre 2005 y $2008^{10}$. Este endeudamiento reducirá la capacidad de maniobra de las entidades en los próximos años, toda vez que trasladará a precios de sus servicios los costes de la financiación.

9 Estos son los costes de mantener la capacidad operativa de la planta sin que necesariamente implique producir un solo metro cúbico de agua desalinizada.

10 De acuerdo a la información contenida en las cuentas anuales de la MCT (Publicadas en el Boletín Oficial del Estado), las deudas a corto plazo con entidades financieras en 2005 se situaban en poco menos de 0,5 millones de $€$, frente a los 78,4 millones de $€$ en 2008; en tanto que las deudas a largo plazo con entidades financieras, que en 2005 eran de 43,5 millones de $€$, en 2008 ascendían a 137,2 millones de $€$. La suma total del endeudamiento con entidades financieras en 2005 era de unos 44 millones de $€$ frente a los 215,6 millones de $€$ de 2008 . 
La segunda pregunta surge del cumplimiento del principio de recuperación de costes emanado de la Directiva Marco del Agua. Las primeras estimaciones realizadas en 2005 establecían un nivel de precios ${ }^{11}$ para ofrecer el agua a los regantes en niveles de $0,36 € / \mathrm{m}^{3}$. Aún a plena capacidad de las plantas desalinizadoras, los costes medios serían de casi tres veces el precio considerado en un principio, lo que lleva a poner en duda el cumplimiento de este postulado de recuperación de costes. Toda vez, si cabe, que a falta de firmar la mayor parte de los convenios para la distribución de los caudales de agua desalinizada, no parece que vayan a emplearse, al menos en el corto plazo, la totalidad de la capacidad de producción de las instalaciones.

\section{CONCLUSIONES}

La desalinización se perfila como el recurso futuro para poder satisfacer las demandas crecientes de agua. Sin embargo, se encuentra condicionada por los costes energéticos y por el consumo de energía.

En la segunda mitad de la primera década del siglo se ha dado un impulso importante a la desalinización en el arco mediterráneo. A través de las actuaciones contempladas en el Programa A.G.U.A. se han puesto en marcha unos 16 proyectos y actuaciones en materia de desalinización. En conjunto, se estima una aportación de nuevos recursos de unos $344,68 \mathrm{hm}^{3}$, de los que unos 155,37 hm $\mathrm{hm}^{3}$ tienen como destino fundamental satisfacer las necesidades de agua de regadíos ubicados en el sureste peninsular.

El coste de estas actuaciones está calculado, en caso de pleno uso de la capacidad productiva de las instalaciones, en algo más de unos 313 millones de Euros anuales, que representa un coste medio de producción de $0,9104 € / \mathrm{m}^{3}\left(0,8634 € / \mathrm{m}^{3}\right.$ si se tienen en cuenta sólo las actuaciones cuya producción tiene como destino el riego agrícola).

Dado el caso que la utilización de la capacidad productiva de las plantas se sitúe en niveles por debajo del $50 \%$, los costes medios se dispararían hasta niveles por encima de los $2-3 € / \mathrm{m}^{3}$, muy alejados de lo que se podría considerar como razonable.

Los costes energéticos suponen una partida importante de estos proyectos alcanzando una proporción cercana al 36\% de los costes totales, de acuerdo a las cifras de diseño con que se estimaron los cálculos hacia el año 2006-2007. Esta partida venía a representar una repercusión de unos 0,3276 € por cada metro cúbico de agua producida.

Con precios de la energía en 2012 muy superiores a los que se aplicaron en los cálculos de los proyectos de las plantas desalinizadoras hacia 2006 en casi un 65\%, el incremento de los costes de producción ha sido notable. Se ha pasado de un coste medio estimado en $2007 \mathrm{de} 0,9104 € / \mathrm{m}^{3}$ a $1,12 € / \mathrm{m}^{3}$ para el conjunto de instalaciones programadas. Este incremento representa algo más de un $23 \%$ en el coste de producción, lo que lleva a alcanzar los costes energéticos niveles de casi el 50\% sobre los costes totales de producción.

Esta situación pone de manifiesto la especial vulnerabilidad de estas actuaciones frente a la energía. Al estimarse un consumo medio de 3,8 $\mathrm{kWh}$ en la producción de $1 \mathrm{~m}^{3}$ de agua, cualquier alteración en el precio de la energía multiplica por ese factor la incidencia sobre el coste medio de producción de esta tipología de recursos.

Como es lógico, la estrategia de diversificación de fuentes de suministro exige tener disponible esta fuente de recursos para cubrir situaciones de demanda que no pueden ser satisfechas con otras fuentes de recursos al no estar disponibles, bien en cantidad o en el tiempo.

No obstante, estrategias diseñadas para satisfacer la demanda basadas exclusivamente en esta fuente de recursos presentan unos riesgos y vulnerabilidades evidentes frente a la incertidumbre en los mercados energéticos. Toda vez que el diseño de esta estrategia ha tenido un marco territorial muy definido, protagonizado por las regiones costeras mediterráneas, lo que representa un lastre para la adecuada flexibilidad de la adaptación de los sistemas productivos de estas regiones. La elevada volatilidad de los precios energéticos pone en evidencia los errores de diseño en la planificación de asignación de recursos llevada a cabo a mediados de la década pasada, como hemos demostrado en el presente trabajo.

11 Caso del Convenio firmado con la Comunidad de Regantes de Águilas para los caudales procedentes de la planta desalinizadora de Águilas/Guadalentín (Murcia). 


\section{RECURSOS ELECTRÓNICOS}

ACUAMED (2006): Estudio de Previsión de Costes del Agua Desalada a Pie de Planta. ACUAMED. Madrid, $33 \mathrm{pp}$.

COMISIÓN EUROPEA (2000): Directiva 2000/60/CE del Parlamento Europeo y del Consejo de 23 de Octubre de 2000 por la que se establece un marco comunitario de actuación en el ámbito de la política de aguas. (DOCE 22.12.2000). Bruselas.

ESTEVAN, A. (2008): Desalación, energía y medio ambiente, en Panel Científico-Técnico de Seguimiento de la Política de Aguas. Ministerio de Medio Ambiente. Madrid, 45 pp.

IZAGIRRE, J. K. (2004): "Técnicas de desalación, costes de construcción y explotación", en Actas de las Jornadas sobre Problemática del agua en la Vega Baja. Guardamar del Segura, pp 167-193.

MARTÍNEZ VICENTE, et al. (2000): "Los costes de la desalación del agua de mar", en Actas del Congreso Nacional sobre Gestión del Agua en cuencas deficitarias. Orihuela, pp. 258-296.

MINISTERIO DE MEDIO AMBIENTE, MEDIO RURAL Y MARINO (2007): Programa A.G.U.A. (Actuaciones para la Gestión y la Utilización del Agua). Madrid, 45 pp.

MINISTERIO DE MEDIO AMBIENTE, MEDIO RURAL Y MARINO (2012): Informes de Viabilidad de Proyectos. Madrid. [http://www.magrama.gob.es/es/agua/planes-y-estrategias/informes-de-viabilidad-deobras-hidraulicas/]. (Consultado: 28/06/2013).

TORRES, M. (2004): "Avances tecnológicos y costes de la desalación”, en Actas de las Jornadas Técnicas sobre la desalación en el Programa AGUA. Grupo Recoletos. Madrid, pp. 23-48.

UNESA (2013): La Industria Eléctrica en 2012. Avance Estadístico. Madrid, pp. 17. 